\title{
FLUTUAÇÃO POPULACIONAL DE Argyrotaenia sphaleropa (MEYRICK, 1909) (LEP: TORTRICIDAE) COM EMPREGO DE FEROMÔNIO SEXUAL SINTÉTICO NA CULTURA DA VIDEIRA ${ }^{1}$
}

\author{
WILSON JOSÉ MORANDI FILHO², MARCOS BOTTON${ }^{2},{\text { ANDERSON DIONEI GRÜTZMACHER }{ }^{4} \text {, SATURNINO NUÑEZ }}^{5}$
}

RESUMO - A lagarta-das-fruteiras, Argyrotaenia sphaleropa (Meyrick, 1909) (Lepidoptera: Tortricidae), é uma espécie freqüentemente encontrada danificando a cultura da videira e outras frutíferas de clima temperado na região Sul do Brasil e no Uruguai. Estudou-se a flutuação populacional de $A$. sphaleropa na cultura da videira com emprego do feromônio sexual sintético. Com base na avaliação semanal de machos de $A$. sphaleropa capturados em armadilha Delta contendo o feromônio sexual sintético (Z11,13-14Ac + Z11,13$14 \mathrm{Al}+\mathrm{Z11}-14 \mathrm{Al}$ na proporção 4:4:1, impregnada em liberadores de borracha na dose de 1.000 $\mu \mathrm{g} /$ septo), durante a safra 2003-2004, foram observados quatro picos populacionais na cultura da videira cultivar Cabernet Sauvignon. O primeiro pico populacional ocorreu no início do mês de outubro, o segundo no início de fevereiro, o terceiro teve o acme em meados do mês de março e o quarto no mês de junho. A temperatura média diária e a precipitação pluviométrica não exerceram influência sobre a captura dos insetos nas armadilhas. As informações deste trabalho permitem direcionar as táticas de controle para os períodos em que a população do inseto é mais elevada nos parreirais.

Termos de indexação: lagarta-das-fruteiras, uva, levantamento populacional

\section{SEASONAL FLUCTUATION OF THE ADULTS OF Argyrotaenia sphaleropa (MEYRICK, 1909) (LEP: TORTRICIDAE) USING SYNTHETIC SEXUAL PHEROMONE IN VINEYARDS}

\begin{abstract}
The South American Tortricid Moth (Meyrick, 1909) (Lepidoptera: Tortricidae) is often found damaging vineyards and other temperate fruit orchards, in Southern Brazil and Uruguay. This work was conducted to study the seasonal occurrence of $A$. sphaleropa adults in vineyards using sexual synthetic pheromone. Based on weekly evaluation of $A$. sphaleropa adults caught in Delta trap baited with synthetic sexual pheromone (Z11, 13-14Ac $+Z 11$, 13-14Al + Z11-14Al in a ratio of 4:4:1, 1000 $\mu \mathrm{g} / \mathrm{septum})$, during 2003/ 2004 season, it was observed four populational peaks in a Cabernet Sauvignon vineyard. The first populational peak occurred in the beginning of October, the second in the beginning of February, the third occurred in middle of March and the forth in June. There was no significative correlation between medium temperature and pluvial precipitation pluvial on adult catches. Information about seasonal fluctuation can be used for grape growers as a tool to guide insecticide treatments for pest control in vineyards.
\end{abstract}

Index Terms- South American Tortricid moth, grape, seasonal fluctuation

\section{INTRODUÇÃO}

A lagarta-das-fruteiras, Argyrotaenia sphaleropa (Meyrick, 1909) (Lep: Tortricidae), tem sido freqüentemente encontrada danificando frutíferas de clima temperado no Sul do Brasil, com destaque para o caquizeiro (Bavaresco, 2004; Manfredi-Coimbra et al., 2005), a pereira (Nora \& Sugiura, 2001), o pessegueiro (Botton et al., 2003a) e a videira (Botton et al., 2003b). Trata-se de uma espécie polífaga, nativa da América do Sul (Meyrick, 1909; Köhler, 1939; Ruffinelli \& Carbonell, 1953; Biezanko, 1961), que também tem sido relatada como praga importante de fruteiras de clima temperado no Uruguai (Nuñez et al., 2002; Bentancourt \& Scatoni, 2006).

$\mathrm{Na}$ cultura da videira, a lagarta de $A$. sphaleropa aloja-se no interior das bagas ainda verdes, danificando a casca do engaço, causando o murchamento e conseqüente queda das uvas (Bentancourt \& Scatoni, 1986). Quando o ataque ocorre próximo à colheita, provoca o rompimento das bagas, resultando no extravasamento do suco sobre o qual proliferam bactérias que ocasionam a podridão ácida, reduzindo a qualidade dos vinhos ou depreciando os cachos para o comércio in natura (Botton et al., 2003b). Além disso, em algumas situações, sobre os ferimentos causados pela alimentação das lagartas, pode ocorrer a proliferação de fungos, como Aspergillus carbonarius, Aspergillus niger e Penicillium sp., responsáveis pela produção da ocratoxina A nos vinhos, com perda da qualidade do produto final, bem como colocando em risco a saúde dos consumidores (Rousseau, 2005).

(Trabalho 145-06). Recebido em:26-09-2006. Aceito para publicação em:16-03-2007

${ }^{2}$ Engenheiro Agrônomo, MSc, Doutorando em Fitossanidade (FAEM), Universidade Federal de Pelotas (UFPel), Câmpus Universitário s/n. C. Postal 354, CEP: 96010-900, Pelotas-RS. E-mail: wilsonmorandi@yahoo.com.br.

${ }^{3}$ Engenheiro Agrônomo, Dr., Pesquisador, Embrapa Uva e Vinho, Rua Livramento 515, C. Postal 130, CEP: 95700-000, Bento Gonçalves-RS. E-mail: marcos@cnpuv.embrapa.br.

${ }^{4}$ Engenheiro Agrônomo, Dr., Prof. Adjunto Departamento de Fitossanidade, FAEM, Universidade Federal de Pelotas (UFPel), Câmpus Universitário s/n.

C. Postal 354, CEP: 96010-900, Pelotas-RS. E-mail: adgrutzm@ufpel.tche.br.

${ }_{5}^{5}$ Engenheiro Agrônomo, Dr. INIA Las Brujas, Apt. 33085, Las Piedras, Canalones, Uruguay. E-mail: snunes@inia.org.uy. 
O controle da lagarta-das-fruteiras, nos últimos anos, tem sido realizado com inseticidas sintéticos, geralmente sem o conhecimento do nível populacional da praga presente nos parreirais (Botton et al., 2003a). Como praticamente não existem informações sobre métodos de controle da espécie nas condições brasileiras, os produtores utilizam inseticidas empregados para outras pragas da videira com base em calendários preestabelecidos, com destaque para o uso do fenitrothion e do triclorfon (Emater/RS, 2003; Bavaresco, 2004). Este manejo está se tornando cada vez mais inviável, e em função do sistema integrado de produção, visa a racionalizar o uso agroquímico, protegendo o ambiente e a saúde dos trabalhadores e consumidores (Naji et al., 2003; Protas \& Sanhueza, 2003).

Entretanto, qualquer método de controle que venha a ser empregado na cultura da videira depende do monitoramento da praga, sendo que sistemas baseados em feromônios sexuais são os mais racionais em termos de execução a campo (Cardé \& Elkinton, 1984). O feromônio sexual da lagarta-das-fruteiras é constituído por quatro componentes: (Z)-11-tetradecenal (Z11 -14A1), e (Z)-11,13-tetradecadienal (Z11,13-14AI); (Z)-11-acetato de tetradecenila (Z11-14Ac), e (Z)-11-13-acetato de tetradecenila (Z11,13-14Ac); os quais ocorrem nas glândulas feromonais na proporção de 1:4:10:40 (Nuñez et al., 2002; Legrand, 2004). No Uruguai, a formulação Z11,13-14Al + Z11-14 Al (9:1), formulada em septos de borracha na dose de $1.000 \mu \mathrm{g} /$ septo, é empregada nos pomares de videira e macieira para o monitoramento de $A$. sphaleropa (Nuñez et al., 2002). Entretanto, no Brasil, essa formulação não foi eficaz, sendo que Bavaresco et al. (2005) demonstraram que a população brasileira de A. sphaleropa responde principalmente aos componentes Z11,13-14Ac + Z11,13-14Al + Z11-14Al (4:4:1), impregnada em liberadores de borracha na dose de $1.000 \mu \mathrm{g} / \mathrm{septo}$, devendo-se repor os liberadores a cada 75 dias.

Neste trabalho, avaliou-se a flutuação populacional de A. sphaleropa na cultura da videira com o emprego do feromônio sexual sintético, visando a definir os picos populacionais da praga na cultura.

\section{MATERIAL E MÉTODOS}

\section{Variação Sazonal de machos de $A$. sphaleropa na cultura da videira}

A flutuação populacional dos adultos de $A$. sphaleropa foi avaliada no período de setembro de 2003 a agosto de 2004, utilizando-se como área de amostragem de dois parreirais de um hectare cada, da cultivar Cabernet Sauvignon, implantados no sistema de espaldeira, no espaçamento de 2,5 x 1,5 m, em 1995 e 2001, localizados no município de Bento Gonçalves- RS (latitude: $24^{\circ} 09^{\prime} 44^{\prime \prime}$ sul, longitude: $51^{\circ} 31^{\prime} 50^{\prime \prime}$ oeste e altitude aproximada de $640 \mathrm{~m})$. Em cada pomar, foi instalada uma armadilha Delta $(10,0 \mathrm{~cm}$ de altura $x 19,5 \mathrm{~cm}$ de largura $x 28,4 \mathrm{~cm}$ de comprimento, com área de fundo adesivo de $385,3 \mathrm{~cm}^{2}$ ), no centro da área experimental, contendo isca com feromônio sexual sintético Z11,13-14Al + Z11,13-14Ac + Z11-14Al impregnado num septo de borracha, na proporção de $4: 4: 1$, na dose de $1.000 \mu \mathrm{g} /$ septo
(Bavaresco et al., 2005). As avaliações foram realizadas semanalmente, contando-se o número de machos capturados, repondo-se o atrativo a cada 75 dias e o fundo de cola sempre que observada perda na adesividade. $O$ feromônio sexual sintético foi fornecido pelo Dr. Saturnino Nunes, do Instituto Nacional de Investigacion Agropecuária (INIA), Uruguai. Nos dois pomares, não foram aplicados inseticidas no período do monitoramento.

A flutuação populacional foi demonstrada graficamente, plotando-se o número médio de machos/armadilha/semana nos dois pomares, em função do tempo. Para análise da flutuação, foram realizadas correlações simples (Pearson) entre o número médio de adultos de $A$. sphaleropa capturados semanalmente com a temperatura média diária $\left({ }^{\circ} \mathrm{C}\right)$ e a precipitação pluviométrica (mm) acumulada sete dias antes da avaliação. Os dados climáticos foram obtidos na estação meteorológica da Embrapa Uva e Vinho de Bento Gonçalves-RS, localizada a 1.000 metros dos parreirais.

\section{RESULTADOS E DISCUSSÃO}

A ocorrência de adultos de $A$. sphaleropa nas armadilhas foi observada durante todo o ano, caracterizando a ausência de estratégias de dormência ou diapausa, conforme relatado por Bentancourt et al. (1988) e Bentancourt \& Scatoni (2006). Durante a safra agrícola de 2003-2004, foram observados quatro picos populacionais de $A$. sphaleropa a partir da brotação da videira, a qual ocorreu em setembro (Figura 1). Os picos populacionais corroboram o trabalho de Manfredi-Coimbra et al. (2001), que estimaram quatro gerações anuais do inseto na região da Serra Gaúcha.

O primeiro pico populacional foi observado no início do mês de outubro sendo este caracterizado por ser a primeira geração do inseto que ocorre quando se encontram disponíveis estruturas vegetativas da videira para alimentação, com destaque para as inflorescências da cultura (Figura 1), o que também foi observado por Bentancourt \& Scatoni (2006) em parreirais do Uruguai.

A segunda geração foi evidenciada pelo aumento na população do inseto a partir do mês de dezembro, com pico populacional no mês de fevereiro. A terceira geração ocorreu nos meses de março a maio, observando-se o acme de captura no final de março. Em hipótese, os insetos da segunda e terceira gerações, por apresentarem maiores níveis populacionais nos pomares, seriam os responsáveis por maiores prejuízos à cultura, principalmente por danificarem diretamente as bagas de uva na fase de maturação.

A quarta geração foi caracterizada pela presença de adultos no período de junho a agosto, com pico em meados de junho. Segundo Bavaresco et al.(2005), esta geração desenvolvese sobre plantas hospedeiras presentes no pomar.

De maneira geral, os picos populacionais observados no período de setembro a julho são semelhantes aos verificados por Bentancourt et al. (1988) e Bentacourt \& Scatoni (2006) na região sul do Uruguai, onde foram observadas quatro gerações anuais, sendo a maior intensidade de danos causada pela segunda e terceira gerações nas culturas da macieira e da videira.

Devido à ausência de um período de diapausa, as gerações 


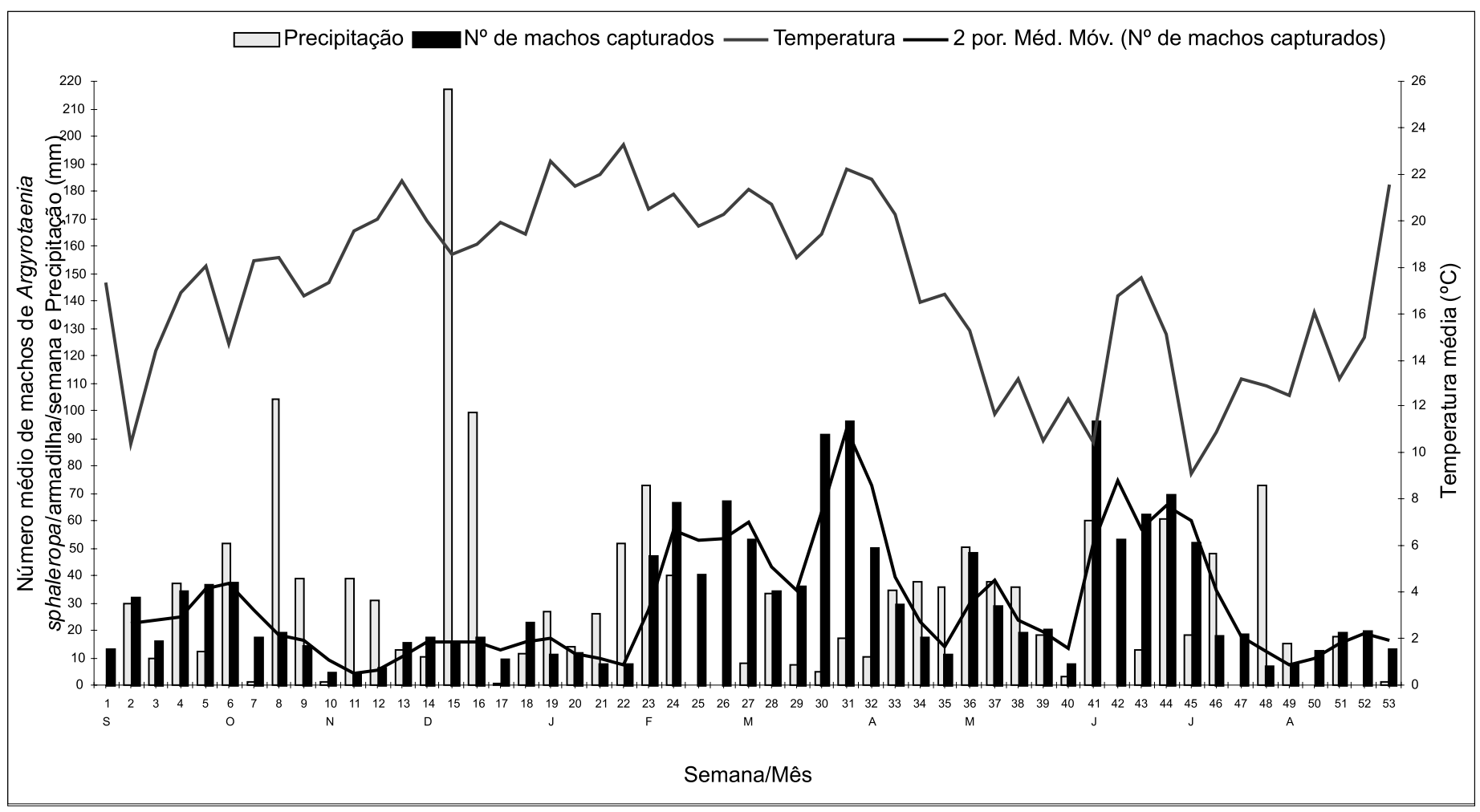

FIGURA 1 - Variação semanal de machos de Argyrotaenia sphaleropa, capturados em armadilhas iscadas com feromônio sexual sintético em vinhedo comercial da cultivar Cabernet Sauvignon. Os valores de temperatura média e precipitação pluviométrica obtidos sete dias anteriores às avaliações, no período de setembro de 2003 a agosto de 2004, foram acumulados. Bento Gonçalves-RS.

ocorrem ininterruptamente ao longo do ano, podendo inclusive haver sobreposição de gerações (Bentancourt et al., 1988; Bentancourt \& Scatoni, 2006).

Através da análise de correlação simples dos fatores climáticos referentes a sete dias anteriores a cada contagem do número de adultos, observou-se que a temperatura média diária e a precipitação pluviométrica não exerceram influência significativa $(\mathrm{Pd} \leq 0,01)$ no número de insetos capturados nas armadilhas, na safra de 2003-2004.

As informações obtidas neste trabalho servem como indicativos para orientar os viticultores da região da Serra Gaúcha quanto aos períodos de maiores níveis populacionais do inseto na cultura, os quais se concentraram no período de fevereiro a março, quando a maioria das cultivares tardia se encontram na fase de colheita. Entretanto, é fundamental que a população do inseto seja monitorada em cada parreiral e sejam conduzidos estudos visando a associar o nível populacional de adultos capturados nas armadilhas e o dano causado pelas lagartas às bagas nas diferentes gerações.

\section{CONCLUSÕES}

Através do emprego de armadilhas com feromônios sexuais, são observados quatro picos populacionais de Argyrotaenia sphaleropa na cultura da videira da cultivar Cabernet Sauvignon, em Bento Gonçalves-RS.

\section{AGRADECIMENTOS}

Parte de trabalho de Dissertação do primeiro autor. Apoio: bolsa de estudos da Coordenação de Aperfeiçoamento de Pessoal de Nível Superior (CAPES) e Conselho Nacional de Desenvolvimento Científico e Tecnológico (CNPq). Bolsista CAPES: Morandi Filho, W.J. Pesquisadores do CNPq: Botton, M.; Grützmacher, A.D. Ao técnico agrícola Odimar Zanuzo Zanardi, pelo auxílio na condução dos experimentos.

\section{REFERÊNCIAS}

BAVARESCO, A. Biologia, comportamento e controle das pragas do caquizeiro Argyrotaenia sphaleropa (Meyrick, 1909) (Lepidoptera: Tortricidae) e Hypocala andremona (Stoll, 1781) (Lepidoptera: Noctuidae). 2004. 110 f. Tese (Doutorado em Fitossanidade) - Faculdade de Agronomia Eliseu Maciel, Universidade Federal de Pelotas, Pelotas-RS, 2004.

BAVARESCO, A.; NUÑEZ, S.; GARCIA, M.S.; BOTTON, M.; SANT'ANA, J. Attraction of males of the South American tortricid moth Argyrotaenia sphaleropa (Meyrick) (Lepidoptera: Tortricidae) to the components of the synthetic sexual pheromone in persimmon. Neotropical Entomology, Londrina, v. 34, n. 4, 2005. 619-625.

BENTANCOURT, C.M.; SCATONI, I.B. Biologia de Argyrotaenia sphaleropa (Meyrick, 1909) (Lep., Tortricidae) en condiciones de laboratório. Revista Brasileira de Biologia, 
São Carlos, v. 46, n.1, p.209-216, 1986.

BENTANCOURT, C.M.; SCATONI, I.B.; NUÑEZ, S. Observaciones sobre la biología de Argyrotaenia sphaleropa (Meyrick, 1909) (Lepidoptera: Tortricidae) em la zona sur del Uruguay. Montevidéo: Universidad de la República, Facultad de Agronomía, 1988. 11 p. (Boletin de Investigación, 13).

BENTANCOURT, C.M.; SCATONI, I.B. Lepidopteros de importancia económica en Uruguay: reconocimiento, biología y daños de las plagas agrícolas y forestales. Montevideo: Hemisfério Sur, 2006. v.1, 437 p.

BIEZANKO, C.M. Olethreutidae, Tortricidae, Phalonidae, Aegeriidae, Glyphipterygidae, Yponomeutidae, Gelechiidae, Oecophoridae, Xylorictidae, Lithocolletidae, Cecidoseidae, Ridiaschinidae, Acrolophidae, Tineidae e Psychidae da zona sudeste do Rio Grande do Sul. Arquivos de Entomologia da Escola de Agronomia “Eliseu Maciel”, Série A, Pelotas, p. $1-16,1961$.

BOTTON, M.; BAVARESCO, A.; GARCIA, M.S. Ocorrência de Argyrotaenia sphaleropa (Meyrick) (Lepidoptera: Tortricidae) danificando pêssegos na Serra Gaúcha, Rio Grande do Sul. Neotropical Entomology, Londrina, v.32, p.503505, 2003a.

BOTTON, M.; AFONSO, A.P.; RINGENBERG, R. Manejo de pragas na cultura da videira. In: SEMINÁRIO INTERESTADUAL DE FRUTICULTURA, 3., 2003, Palmas. Anais... Palmas-PR: FACIPAL, 2003 b. p.23-31.

CARDÉ, R.T.; ELKINTON, J.S. Field trapping with attractants: methods and interpretation. In: HUMMEL, H. E.; MILLER, T. A. (Ed.). Techniques in pheromone research. New York: Springer-Verlag, 1984. p. 111-129.

EMATER/RS. Recomendações para o manejo das doenças fúngicas e insetos pragas da videira. Porto Alegre: EMATER/ RS; Bento Gonçalves: Embrapa Uva e Vinho, 2003. 72 p.

HAJI, F. N. P.; LOPES, P. R. C.; MOREIRA, A. N.; COSTA, V. S. de $O$.(Ed.). Normas técnicas e documentos de acompanhamento da produção integrada de uvas finas de mesa. Petrolina-PE : Embrapa Semi-Árido, 2003. 72 p. (Documentos, 184).

KÖHLER, P. Tres nuevos microlepidópteros argentinos. Anales de la Sociedad Científica Argentína, Buenos Aires, v.128, p.369-374, 1939.

KOVALESKI, A.; RIBEIRO, L.G. Manejo de pragas na produção integrada de maçã. In: PROTAS, J. F.da. S.; SANHUEZA, R. M. V. Produção integrada de frutas: o caso da maçã no Brasil. Bento Gonçalves: Embrapa Uva e Vinho, 2003. p.61-68.

LEGRAND, S.; BOTTON, M.; CORACINI, M.; WITZGALL, P.; UNELIUS, C.R. Synthesis and field tests of sex pheromone components of the leafroller Argyrotaenia sphaleropa. Zeitschrift für Naturforschung, Journal of Biosciences, Bangalore, v. 9/10, p. 708-712, 2004.

MANFREDI-COIMBRA, S.; GARCIA, M.S.; BOTTON, M. Thermal Requirements and Estimate of the Number of Generations of Argyrotaenia sphaleropa (Meyrick) (Lepidoptera: Tortricidae). Neotropical Entomology, Londrina, v. 30, n. 4, p. 553-557, 2001.
MANFREDI-COIMBRA, S.M.; GARCIA, M.S.; LOECK, A.E.; BOTTON, M.; FORESTI, J. Aspectos biológicos de Argyrotaenia sphaleropa (Meyrick, 1909) (Lepidoptera:Tortricidae) em dietas artificiais com diferentes fontes protéicas. Ciência Rural, Santa Maria, v.35, n.2, p.259265, 2005.

MEYRICK, E. Description of Microlepidoptera from Bolívia and Peru. The Transactions of the Royal Entomological Society of London, London, v.15, p.13-43, 1909.

NORA, I.; SUGIURA, T. Estudo da entomofauna associada à cultura de pereiras japonesas (Housui, Kousui e Nijisseiki), em Santa Catarina, Brasil e técnicas de manejo. In: ENCONTRO NACIONAL DE FRUTICULTURA DE CLIMA TEMPERADO, 4., 2001, Fraiburgo. Anais... Caçador-SC: Epagri, 2001.p. 164.

NUÑEZ, S.;VLIEGER, J.J.; RODRIQUEZ, J.J.; PERSOONS, C.J.; SCATONI, I. Sex pheromone of South American tortricid moth Argyrotaenia sphaleropa. Journal of Chemical Ecology, New York, v.28, p.425-432, 2002.

PROTAS, J.F. da S.; SANHUEZA. R.M.V. Produção integrada de frutas: o caso da maçã no Brasil. Bento Gonçalves: Embrapa Uva e Vinho, 2003. 192 p.

ROUSSEAU, J. Ochratoxin A in wines: current knowledges. Disponível em: <http://www.icv.fr/kiosqueuk/refs/ vinideaOTAenglish1.pdf $>$. Acesso em: 17 mar. 2005.

RUFFINELLI, A.; CARBONELL, C. Segunda lista de insectos y otros artópodos de importância económica en el Uruguay. Revista de la Asociación de Ingenieros Agrónomos, Montevidéu, v.94, p.33-82, 1953. 